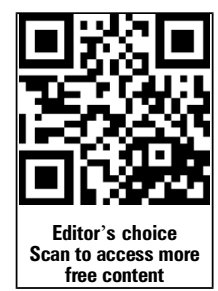

- Additional material is published online only. To view please visit the journal online (http://dx.doi.org/10.1136/ neurintsurg-2015-011686).

${ }^{1} \mathrm{MRI}$ Core, Houston Methodist Research Institute, Houston, Texas, USA

${ }^{2}$ Cerebrovascular Center, Houston Methodist Hospital, Houston, Texas, USA ${ }^{3}$ Department of Neurosurgery, Houston Methodist Hospital, Houston, Texas, USA

\section{Correspondence to} Dr J R Anderson, Houston Methodist Research Institute, 6670 Bertner Ave, R1-303, Houston, TX 77030, USA; jranderson@houstonmethodist. org

Received 5 February 2015 Revised 19 March 2015 Accepted 23 March 2015 Published Online First 10 April 2015

\title{
Three-dimensional printing of anatomically accurate, patient specific intracranial aneurysm models
}

\author{
Jeff R Anderson, ${ }^{1}$ Walker L Thompson, ${ }^{1}$ Abdulaziz K Alkattan, ${ }^{1}$ Orlando Diaz, ${ }^{2,3}$ \\ Richard Klucznik, ${ }^{2,3}$ Yi J Zhang, 2,3 Gavin W Britz, ${ }^{2,3}$ Robert G Grossman, ${ }^{3}$ \\ Christof Karmonik ${ }^{1,2,3}$
}

\section{ABSTRACT}

Objective To develop and validate a method for creating realistic, patient specific replicas of cerebral aneurysms by means of fused deposition modeling. Methods The luminal boundaries of 10 cerebral aneurysms, together with adjacent proximal and distal sections of the parent artery, were segmented based on DSA images, and corresponding virtual three-

dimensional (3D) surface reconstructions were created. From these, polylactic acid and MakerBot Flexible Filament replicas of each aneurysm were created by means of fused deposition modeling. The accuracy of the replicas was assessed by quantifying statistical significance in the variations of their inner dimensions relative to 3D DSA images. Feasibility for using these replicas as flow phantoms in combination with phase contrast MRI was demonstrated.

Results 3D printed aneurysm models were created for all 10 subjects. Good agreement was seen between the models and the source anatomy. Aneurysm diameter measurements of the printed models and source images correlated well $(r=0.999 ; p<0.001)$, with no statistically significant group difference $(p=0.4)$ or observed bias. The SDs of the measurements were $0.5 \mathrm{~mm}$ and $0.2 \mathrm{~mm}$ for source images and 3D models, respectively. 3D printed models could be imaged with flow via MRI.

Conclusions The 3D printed aneurysm models presented were accurate and were able to be produced inhouse. These models can be used for previously cited applications, but their anatomical accuracy also enables their use as MRI flow phantoms for comparison with ongoing studies of computational fluid dynamics. Proof of principle imaging experiments confirm MRI flow phantom utility.

\section{INTRODUCTION}

Hemodynamics in cerebral aneurysms has been investigated in respect of its potential for assessing aneurysm rupture risk, growth, or recanalization. ${ }^{1-5}$ Standard of care imaging protocols, employing CT angiography and DSA, currently do not allow measurement or quantification of blood velocity or volumetric flow rates, such as are available by phase contrast MRI (pcMRI) or Doppler ultrasound. ${ }^{6-8}$ Implementation of additional imaging examinations is a subject of research studies and may be undesirable if treatment of the patient is unduly delayed. As a surrogate technique, computational modeling has been proposed to study aneurysm hemodynamics, but the validity and applicability of its results are yet to be established. Alternatively, ex vivo imaging of aneurysm replicas, using either simplified or patient specific geometries, has been proposed. To date, creating these replicas has been associated with increased expense in terms of time and money, and thereby they are only practical for a few selected aneurysms in a dedicated research environment. ${ }^{9-13}$

An alternative approach for creating aneurysm replicas has recently become available with the introduction of a new generation of personal threedimensional (3D) printers as a consumer market product. ${ }^{14}$ Additive manufacturing techniques, such as $3 \mathrm{D}$ printing, have been applied to various medical applications, ${ }^{15}$ such as creating accurate models of individual patient anatomy for patient education, ${ }^{16}{ }^{17}$ as visual aids during surgery ${ }^{18}$ and for surgery training/planning, ${ }^{19-21}$ and as patient specific phantoms. ${ }^{22}$ The new generation of consumer grade $3 \mathrm{D}$ printers now enables the entire $3 \mathrm{D}$ printing process to be onsite, fast, and cost effective. However, the accuracy of the replicas created in this manner remains to be established.

In this study, we quantified variations in the luminal dimensions of replicas of cerebral aneurysms created by this new technique, by comparing the inner dimensions with source DSA images. We further demonstrated the feasibility of utilizing these replicas as flow phantoms for studying aneurysm hemodynamics ex vivo within a flow loop by means of pcMRI. These experiments serve to establish a way of validating results from computational simulations, but also of optimizing pcMRI imaging techniques (ie, minimizing the duration of imaging examinations in order to avoid interference with the standard treatment schedule).

\section{MATERIALS AND METHODS Image post-processing}

Local institutional review board approval was obtained for this retrospective study. Threedimensional DSA image data from diagnostic angiographic imaging examinations (Siemens Axiom Artis; Siemens AG, Munich, Germany) of 10 patients were retrospectively collected. Images were anonymized and exported in standard DICOM format to a dedicated workstation (quad-core MacBook Pro; Apple, Cupertino, California, USA). The following postprocessing steps were carried out with Image $(\mathrm{NIH}$, Bethesda, Maryland, USA). 
1. Images were cropped around the aneurysm region of interest and voxel size adjusted to $0.13 \mathrm{~mm}$ isotropic by bicubic interpolation.

2. Background noise below a threshold value, obtained via the Otsu method which minimizes intra-class variance between two groups, was excluded. ${ }^{23}$

3. The luminal boundary was segmented using the histogram based Intermodes method. ${ }^{24}$ Small vessels were removed from the parent artery and the resultant image stack was filtered with respect to $3 \mathrm{D}$ connectivity ('find connected regions' plugin; ImageJ ${ }^{25}$ to select only the parent artery and aneurysm.

The segmented vasculature was dilated by four iterations/ voxels to account for the wall thickness of the 3D printed replicas. The dataset was exported from Image $J$ in the visualization toolkit (VTK) format.

The VTK file was imported into paraview (Kitware Inc, Clifton Park, New York, USA) and a contour filter was applied to the data to build an isosurface (ie, 3D surface model) of the artery and aneurysm with the boundary of the isosurface dictated by the segmentation results. A smoothing filter was applied to the isosurface to remove rough luminal surfaces that were artifacts of imaging/segmentation. The resultant 3D model was stored as a stereolithogray (STL) file.

\section{Fused deposition modeling}

Fused deposition modeling (FDM) is realized by passing a plastic with a relatively low melting point through an extruding tip. The xy location of the tip is manipulated while a fine filament of molten plastic is extruded. The extruded filament quickly solidifies to create a single slice of a $3 \mathrm{D}$ model. In this way, a 3D printed model can be made layer by layer as the process is iterated at multiple heights/z locations. All 3D printing was carried out with a MakerBot Replicator 2 3D printer (MakerBot Industries, LLC, Brooklyn, New York, USA).

\section{Wall thickness calibration}

Various test calibration cubes (dimensions $2 \mathrm{~cm} \times 2 \mathrm{~cm} \times 1 \mathrm{~cm}$ ) were printed and halted halfway through the printing process. Shell thicknesses of the calibration cubes were measured on each of the four vertical walls using calipers. Measurements were made for calibration cubes with number of printed shells equal to 1-3 and for calibration cubes printed in two materials, polylactic acid and MakerBot Flexible Filament. Wall thickness measurements were modeled by linear regression to establish a relationship between number of printed wall shells $S$ and actual wall thickness T.

Three-dimensional printing of aneurysm replicas

Replicas were printed in both polylactic acid (rigid walls) and Makerbot Flexible Filament (flexible walls). For further details, including printing parameters, see the online supplementary appendix A.1. An infill of $0 \%$ was used to obtain hollow interiors. Following printing, the rafts, supports, and capped vessel ends were removed from the models.

\section{Measurement of luminal boundaries in the replicas}

Three independent operators measured the longest dimension of each aneurysm in both the source images and the replicas for assessing the accuracy of the inner diameter with respect to the imaged aneurysmal lumen. Printed models were cut open with a small saw to expose the interior of the aneurysm replica. Calipers were used for the measurements. In parallel, projection images were constructed from the unsegmented source images (360 images in increments of $1^{\circ}$ ). The image stack was rotated to highlight the longest dimension, which was then measured in ImageJ.

\section{MRI flow phantom testing}

Replicas were prepared for use as MRI flow phantoms as follows. Capped ends of the printed vessels were removed, and tubing was attached to the proximal and distal parent artery segments. A thin synthetic rubber coating was applied onto the surface of the replicas for waterproofing (Plasti Dip; Plasti Dip International, Blaine, Minnesota, USA). Tubing at the parent artery segments was attached to a flow loop consisting of a continuous flow pump located in the MRI control room and tubing stretching from the control room to the MRI scanner through a communicating waveguide. Aneurysm flow phantoms were filled with water containing $0.2 \mathrm{mM}$ gadopentetate dimeglumine (Magnevist; Bayer Inc, Toronto, Ontario, Canada) and visually inspected for leaks. MRI examinations were carried out on a clinical scanner (3 T Philips Ingenia; Philips Healthcare, Best, The Netherlands) with an eight channel wrist coil. T1 weighted images were acquired without flow. Proof of principle, high resolution quantitative flow images were acquired using 3D QFlow while the contrast agent/water solution was circulated continuously through the replicas. For acquisition parameters, please see the online supplementary appendix A.2. Three QFlow images were acquired per aneurysm to encode flow on all three primary axes. Flow data were imported into paraview and visualized using vectors denoting strength and direction of flow.

\section{RESULTS}

\section{Fused deposition modeling}

Wall thickness calibration

Shell thickness measurements were carried out prior to the segmentation and printing of the replicas. The results (ie, a single shell thickness corresponding to $0.46 \mathrm{~mm}$ ) could be translated into the number of dilations $(n=4)$ in the segmentation step as one pixel was equivalent to a length of $0.13 \mathrm{~mm}$. For further details, including printing parameters, see the online supplementary appendix A.3.

\section{Three-dimensional printing of aneurysm replicas}

Visual inspection showed good agreement between the virtual 3D luminal surface reconstruction and the printed replicas (figure 1). Comparison of luminal measurements from both the DSA source images and the printed replicas showed good agreement (table 1, figures 1 and 2). The average inter-operator SD for the caliper measurement (ie, the physical measurement of the replicas) was $0.2 \mathrm{~mm}$. This is comparable with the average $\mathrm{SD}$ of $0.5 \mathrm{~mm}$ for the digital measurement (ie, the virtual measurement in Image J of the lumen in the DSA source images). The two measurements correlated very well, with a correlation coefficient (r) of 0.999 and a $\mathrm{p}$ value $<0.001$ for the correlation. Bland-Altman plots of the results did not show an apparent bias in the measurements (figure 2). No statistically significant difference between the physical and virtual measurements was found $(p=0.4$ from a two tailed paired $t$ test $)$.

\section{MRI flow phantom testing}

T1 weighted images reproduced the morphology and geometry of the replicas (figure 2). Bubbles detected in these images were removed by manual agitation before performing the pcMRI imaging. Flow vectors reconstructed from the high resolution 3D QFlow images succeeded in reproducing well know flow features in cerebral aneurysms (recirculating flow in the aneurysm dome, figure 2). 


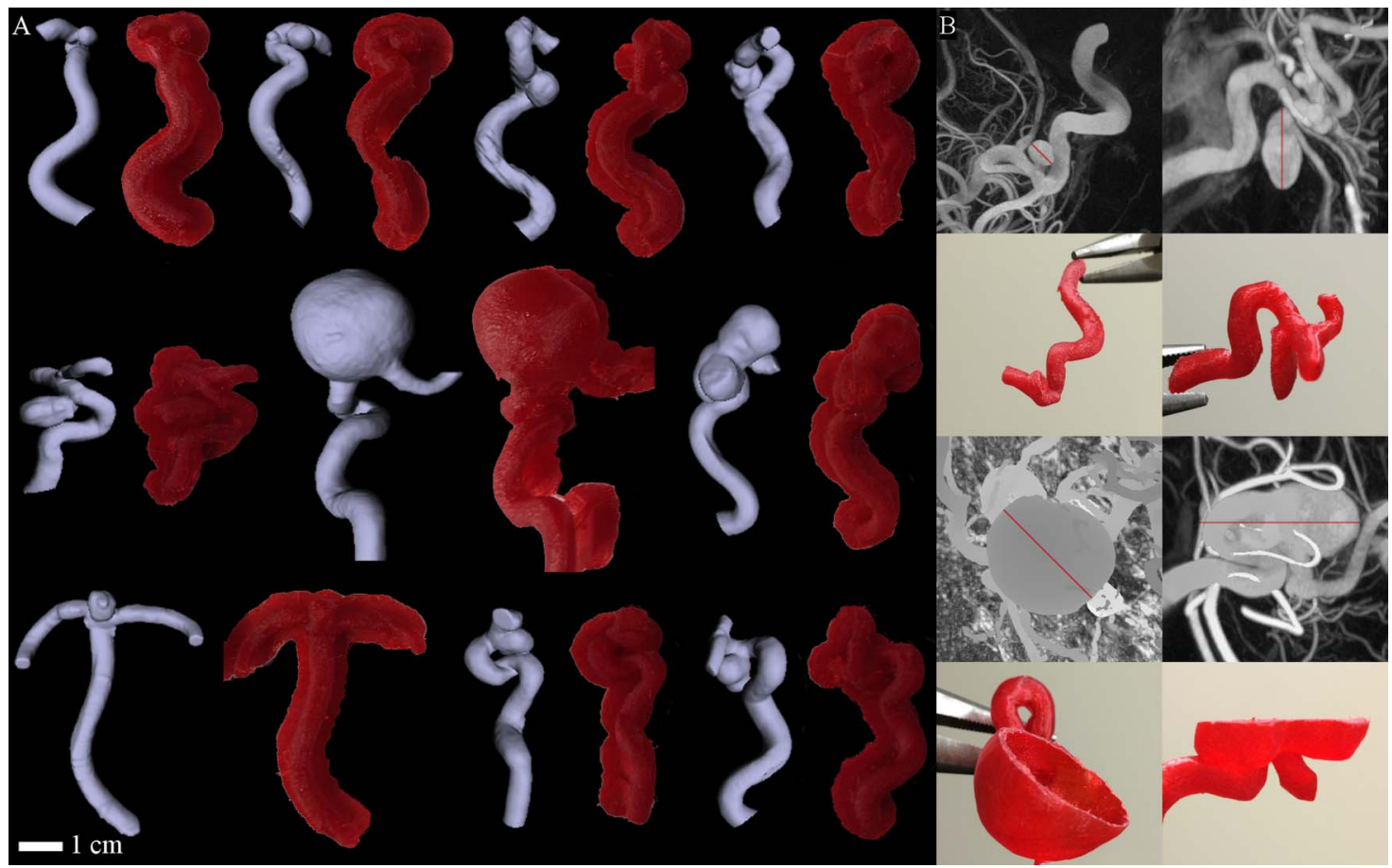

Figure 1 (A) Surface reconstructions and corresponding replicas for all 10 aneurysms. (B) Four representative maximum intensity projections of DSA images, with the aneurysm diameter highlighted in red, and corresponding replicas that have been cut open for measurement of the aneurysm diameter of the replicas.

\section{DISCUSSION}

A prerequisite for the use of patient specific, anatomically accurate $3 \mathrm{D}$ replicas of human anatomy require fast and cost effective manufacturing methods that, at the same time, are also accurate. As we have demonstrated for cerebral aneurysms, FDM or 3D printing with consumer grade $3 \mathrm{D}$ printers can fulfill these requirements.

Alternative additive manufacturing methods to FDM exist and include selective laser sintering (SLS) and stereolithography (SLA). SLS has finer resolution than FDM and larger models can be produced. However, no consumer grade SLS printers currently exist. SLA printers also have finer resolution than FDM printers, and a small number of consumer grade SLA printers are available. A trade off of consumer grade SLA printers versus professional grade SLA printers is that the build volume is much smaller. A disadvantage of SLA compared with FDM is that consumables are more expensive and additional curing

Table 1 Measurement results for the aneurysms

\begin{tabular}{|c|c|c|c|c|}
\hline \multirow[b]{2}{*}{ Aneurysm } & \multicolumn{2}{|l|}{ DSA Images } & \multicolumn{2}{|l|}{ Replicas } \\
\hline & $\begin{array}{l}\text { Longest dimension } \\
\text { (average) }(\mathrm{mm})\end{array}$ & SD & $\begin{array}{l}\text { Longest dimension } \\
\text { (average) }(\mathrm{mm})\end{array}$ & SD \\
\hline A & 3.6 & 0.5 & 3.3 & 0.2 \\
\hline B & 4.8 & 0.2 & 4.9 & 0.3 \\
\hline C & 6.8 & 0.2 & 6.9 & 0.1 \\
\hline D & 7.8 & 0.6 & 6.9 & 0.3 \\
\hline $\mathrm{E}$ & 11.8 & 0.6 & 11.5 & 0.3 \\
\hline $\mathrm{F}$ & 26.6 & 1.1 & 26.8 & 0.2 \\
\hline G & 23.9 & 0.4 & 24.2 & 0.1 \\
\hline H & 7.5 & 0.7 & 7.85 & 0.07 \\
\hline I & 7.8 & 0.8 & 7.3 & 0.5 \\
\hline J & 11.4 & 0.2 & 11.1 & 0.3 \\
\hline
\end{tabular}

steps are required following printout. The resolution of the FDM printer used, although coarser than SLS and SLA printers, was found to be sufficient for the models at hand.

Several additive manufacturing references for aneurysm models have previously been reported. ${ }^{17} 18212627$ In contrast with the technique described here, these models used professional grade SLA printers. Furthermore, the created replicas were not hollow, limiting their direct use as flow phantoms. An alternative is the use of silicon models manufactured to accurately reproduce patient anatomy. ${ }^{28} 29$ The manufacture of such models involves casting, a multiple step technique that starts with a solid object that can be used to make the initial mold. Solid (ie, non-hollow) additive manufactured models as well as excised epoxy filled cadaveric vasculature have been used as this initial casting object. ${ }^{10-12}$ Due to the complex manufacturing process, studies performed with these models only used a relatively small number of cases where the actual models were not created inhouse but purchased from a vendor. ${ }^{9-13} 22$

One potential application of these replicas is their use as MRI flow phantoms with the goal of establishing an imaging protocol for visualizing and quantifying aneurysm hemodynamics. MRI flow measurement results ${ }^{12}{ }^{30}$ can then be compared with computational fluid dynamics modeling for validation ${ }^{31} 32$ or used directly to characterize a particular aneurysm. The proof of principle images we acquired shows that the replicas created with consumer grade $3 \mathrm{D}$ printers and modified accordingly are adequate for this purpose. It should be noted that computational fluid dynamics simulations, by design, are an approximation of a real system. Dependent on the detail of the approximation, the computed solution will be more accurate (ie, closer to the realistic behavior of aneurysmal hemodynamics). Wall compliancy depends on the material properties of the aneurysm wall and might be affected by local collagen deficiencies or other disease. As such, it is inherently difficult to incorporate these aneurysm specific variations into computational 

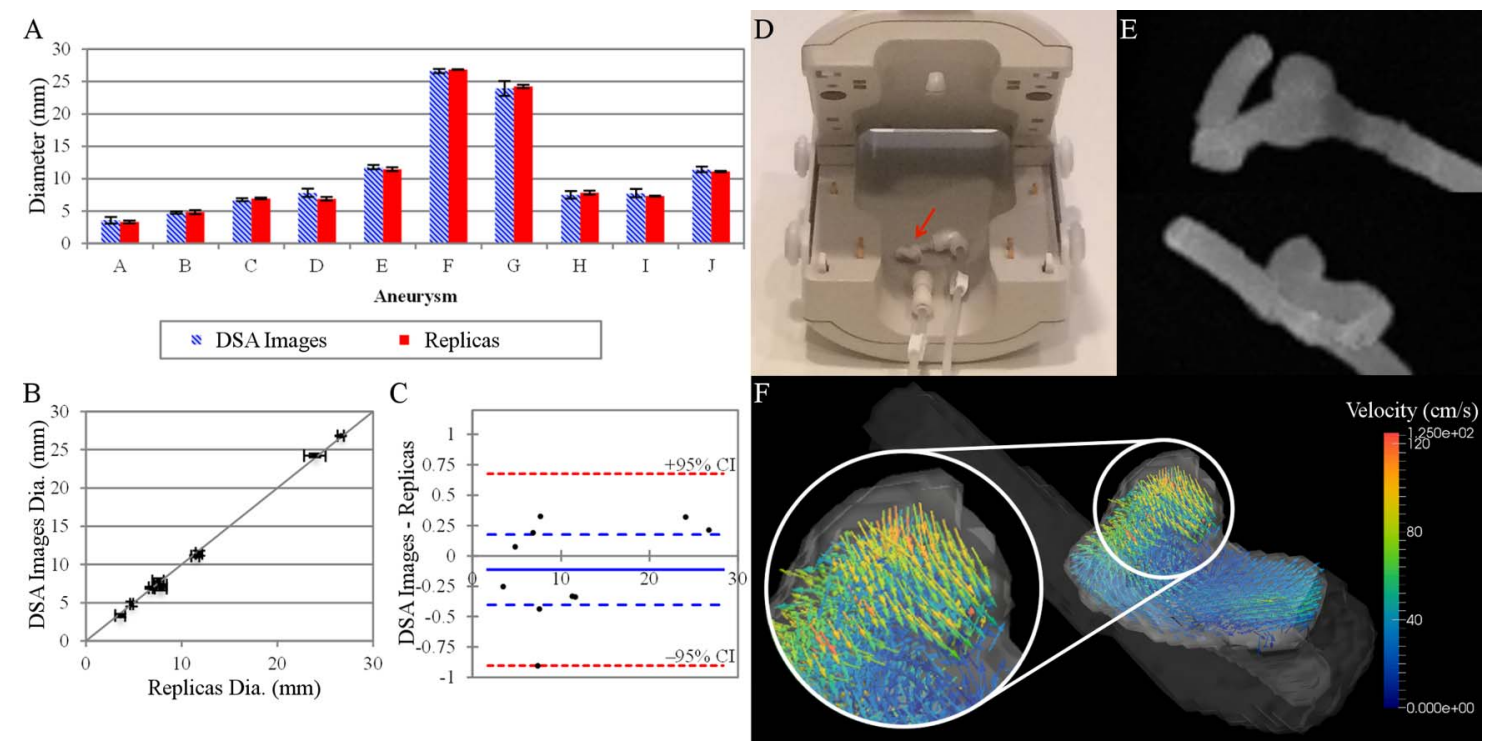

Figure 2 (A) Measurement results of the aneurysms and aneurysm replicas; the virtual measurement results of the luminal boundary in the source DSA images and the caliper measurements of the replicas are shown $( \pm S D)$. (B) Correlation between the two measurement methods $(p<0.001)$. (C) Corresponding Bland-Altman plot for (A) and (B); $95 \%$ Cls are shown (red), the bias (solid line), and $95 \%$ Cls of the bias (blue). (D) MRI scanner setup of the aneurysm phantom (arrow) in the wrist coil. (E) Maximum intensity projections of T1 weighted MRI images for a representative MRI flow phantom from the front (top) and side (bottom). (F) Quantitative flow vector image (side perspective) highlighting the flow patterns for aneurysm replica shown in (E); arrowheads denote flow direction and color denotes flow velocity (magnified in the inset).

methods. Matching aneurysm wall dynamics to imaged dynamics in aneurysm replicas might provide a potential remedy. In any case, wall compliance most likely will have an effect on wall shear stress and related quantities, ${ }^{33}$ and the order of the effect will directly depend on the degree of wall motion-that is, strongly pulsating blebs will show the largest variations from computed simulations assuming rigid walls. Nevertheless, models with a rigid wall (virtual and/or 3D printed) are assumed to provide an approximation for aneurysmal hemodynamics and imaging rigid 3D models can serve as a direct validation of computational fluid dynamics results for rigid computational models.

Performing MRI measurements ex vivo, as opposed to imaging of the same anatomy in vivo, affords an unique opportunity. One advantage consists in achieving higher spatial and temporal resolution (and/or improved signal to noise ratio) as time is less of a constraint and motion artifacts are non-existent. That said, the results of such experiments, due to the anatomical exactness of the MRI phantoms, are expected to foster a better understanding of the lower resolution in vivo MRI measurements. Other potential applications, due to the anatomical exactness of the replicas, may be as an aid to better visualizing the vascular pathology in question and to assist in facilitating presurgical planning. Future research efforts will include perfecting current methods to make the aneurysm replicas and MRI flow data even more realistic and also pulsatile pumping of solutions with viscosity matched to that of blood and more compliant printed vessel walls. An important first step to incorporating wall elasticity into the models has been presented by printing replicas in MakerBot Flexible Filament. Even in the absence of compliant vessel walls, however, the phantoms created will make it possible to quickly develop and optimize imaging protocols for a particular aneurysm (patient) without the patient having to be subjected to lengthy optimization scans inside the scanner room that potentially can compromise patient comfort and/or safety.

\section{CONCLUSION}

Anatomically accurate replicas of cerebral aneurysms were created by a consumer grade 3D printer in a fast and cost effective manner, promoting their routine inhouse use. Potential applications include creating realistic flow phantoms or providing advanced visualizations of these vascular pathologies for facilitating presurgical planning.

\section{Twitter Follow Abdulaziz Alkattan at @azizkat}

Contributors JRA and CK designed the study and are guarantors. JRA carried out the study, acquired ex vivo data, analyzed the data, and drafted the manuscript. WLT and AKA analyzed the data. OD, RK, YJZ, and GWB acquired the in vivo data. RGG and CK critically revised the manuscript. All authors reviewed the manuscript.

Competing interests None.

Ethics approval The study was approved by Houston Methodist Institutional Review Board.

Provenance and peer review Not commissioned; externally peer reviewed.

\section{REFERENCES}

1 Cebral JR, Vazquez M, Sforza DM, et al. Analysis of hemodynamics and wall mechanics at sites of cerebral aneurysm rupture. J Neurointerv Surg Published Online First: 14 May 2014.

2 Schnell S, Ansari SA, Vakil P, et al. Three-dimensional hemodynamics in intracranial aneurysms: influence of size and morphology. J Magn Reson Imaging 2014;39:120-31.

3 Levitt MR, McGah PM, Aliseda A, et al. Cerebral aneurysms treated with flow-diverting stents: computational models with intravascular blood flow measurements. AJNR Am J Neuroradiol 2014;35:143-8.

4 Chien A, Sayre J. Morphologic and hemodynamic risk factors in ruptured aneurysms imaged before and after rupture. AJNR Am J Neuroradiol 2014;35:2130-5.

5 Karmonik C, Diaz O, Klucznik R, et al. Quantitative comparison of hemodynamic parameters from steady and transient CFD simulations in cerebral aneurysms with focus on the aneurysm ostium. J Neurointerv Surg Published Online First: 10 Apr 2014.

6 Ansari SA, Schnell S, Carroll T, et al. Intracranial 4D flow MRI: toward individualized assessment of arteriovenous malformation hemodynamics and treatment-induced changes. AJNR Am J Neuroradiol 2013;34:1922-8.

7 Futami K, Sano H, Misaki K, et al. Identification of the inflow zone of unruptured cerebral aneurysms: comparison of 4D flow MRI and 3D TOF MRA data. AJNR Am J Neuroradiol 2014;35:1363-70. 
8 Karmonik C, Zhang YJ, Diaz 0 , et al. Magnetic resonance imaging as a tool to assess reliability in simulating hemodynamics in cerebral aneurysms with a dedicated computational fluid dynamics prototype: preliminary results. Cardiovasc Diagn Ther 2014:4:207-12.

9 Costalat V, Lebars E, Sarry L, et al. In vitro evaluation of 2D-digital subtraction angiography versus 3D-time-of-flight in assessment of intracranial cerebral aneurysm filling after endovascular therapy. AJNR Am J Neuroradiol 2006;27:177-84.

10 Goubergrits L, Thamsen B, Berthe A, et al. In vitro study of near-wall flow in a cerebral aneurysm model with and without coils. AJNR Am J Neuroradiol 2010:31:1521-8.

11 Imbesi SG, Knox K, Kerber CW. Aneurysm flow dynamics: alterations of slipstream flow for neuroendovascular treatment with liquid embolic agents. AJNR Am J Neuroradiol 2003:24:2044-9.

12 Isoda $\mathrm{H}$, Hirano $\mathrm{M}$, Takeda $\mathrm{H}$, et al. Visualization of hemodynamics in a silicon aneurysm model using time-resolved, 3D, phase-contrast MRI. AJNR Am J Neuroradiol 2006;27:1119-22.

13 Piotin $M$, lijima $A$, Wada $H$, et al. Increasing the packing of small aneurysms with complex-shaped coils: an in vitro study. AJNR Am J Neuroradiol 2003;24:1446-8.

14 A view from inside the 3D manufacturing movement. http://www.washingtonpost. com/blogs/innovations/post/a-view-from-inside-the-3d-manufacturing-movement/ 2010/12/20/gIQAVYZCgR_blog.html (accessed 30 May 2014).

15 Rengier $F$, Mehndiratta A, von Tengg-Kobligk H, et al. 3D printing based on imaging data: review of medical applications. Int J Comput Assist Radio/ Surg 2010;5:335-41.

16 Noecker AM, Chen JF, Zhou Q, et al. Development of patient-specific three-dimensional pediatric cardiac models. ASAIO J 2006;52:349-53.

17 Opolski AC, Erbano BO, Schio NA, et al. Experimental three-dimensional biomodel of complex aortic aneurysms by rapid prototyping technology. 3D Print Addit Manufact 2014;1:88-94.

18 Wurm G, Tomancok B, Pogady P, et al. Cerebrovascular stereolithographic biomodeling for aneurysm surgery. Technical note. J Neurosurg 2004;100:139-45.

19 Mashiko T, Otani K, Kawano R, et al. Development of three-dimensional hollow elastic model for cerebral aneurysm clipping simulation enabling rapid and low cost prototyping. World Neurosurg 2013;83:351-61.

20 Kimura T, Morita A, Nishimura K, et al. Simulation of and training for cerebral aneurysm clipping with 3-dimensional models. Neurosurgery 2009;65:719-25; discussion 725-716.
21 Khan IS, Kelly PD, Singer RJ. Prototyping of cerebral vasculature physical models. Surg Neurol Int 2014;5:11.

22 Mordasini P, Kraehenbuehl AK, Byrne JV, et al. In vitro and in vivo imaging characteristics assessment of polymeric coils compared with standard platinum coils for the treatment of intracranial aneurysms. AJNR Am J Neuroradiol 2013;34:2177-83.

23 Otsu N. A threshold selection method from gray-level histograms. Syst Man Cybern IEEE Trans 1979:9:62-6.

24 Prewitt JMS, Mendelsohn ML. The analysis of cell images. Ann N Y Acad Sci 1966;128:1035-53.

25 Schindelin J, Arganda-Carreras I, Frise E, et al. Fiji: an open-source platform for biological-image analysis. Nat Methods 2012;9:676-82.

26 Erbano BO, Opolski AC, Olandoski M, et al. Rapid prototyping of three-dimensional biomodels as an adjuvant in the surgical planning for intracranial aneurysms. Acta Cir Bras 2013:28:756-61.

27 D'Urso PS, Thompson RG, Atkinson RL, et al. Cerebrovascular biomodelling: a technical note. Surg Neurol 1999;52:490-500.

28 Liepsch D, Zimmer R. [A method for the preparation of true-to-scale inflexible and natural elastic human arteries (author's transl)]. Biomed Tech (Berl) 1978;23:227-30

29 Chueh JY, Wakhloo AK, Gounis MJ. Neurovascular modeling: small-batch manufacturing of silicone vascular replicas. AJNR Am J Neuroradiol 2009;30:1159-64.

30 van Ooij P, Schneiders JJ, Marquering HA, et al. 3D cine phase-contrast MRI at 3T in intracranial aneurysms compared with patient-specific computational fluid dynamics. AJNR Am J Neuroradiol 2013;34:1785-91.

31 Ford MD, Nikolov HN, Milner JS, et al. PIV-measured versus CFD-predicted flow dynamics in anatomically realistic cerebral aneurysm models. J Biomech Eng 2008;130:021015.

32 Anderson JR, Diaz O, Klucznik R, et al. Validation of computational fluid dynamics methods with anatomically exact, 3D printed MRI phantoms and 4D pcMRI. Conf Proc IEEE Eng Med Biol Soc 2014;2014:6699-701.

33 Valencia A, Muñoz F, Araya $S$, et al. Comparison between computational fluid dynamics, fluid-structure interaction and computational structural dynamics predictions of flow-induced wall mechanics in an anatomically realistic cerebral aneurysm model. Int J Comput Fluid Dyn 2009;23:649-66.

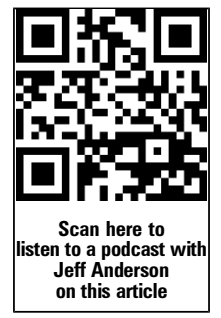

